\title{
Feasibility of Body-Centric Systems Using Passive Textile RFID Tags
}

\author{
S. Manzari, C. Occhiuzzi, and G. Marrocco \\ DISP - University of Roma Tor Vergata \\ Via del Politecnico, 1, 00133, Rome, Italy \\ E-mail: sabinamanzari@virgilio.it; occhiuzzi@disp.uniroma2.it; marrocco@disp.uniroma2.it
}

\begin{abstract}
Recent progresses in the design of wearable RFID-tag antennas stimulate the idea of passive body-centric systems, wherein the required power to drive the wearable tags is directly scavenged from the interrogation signal emitted by the reader unit. While active body-centric links have been extensively investigated, the feasibility of passive systems is still questionable, due to the poor sensitivity of the tags and due to the modest reading distances. This paper describes a systematic measurement campaign involving low-profile wearable textile tags in the UHF RFID band. It was demonstrated that both on-body and off-body links are affordable, with a power budget fully compliant with the available technology and the safety standards. The experiments permitted identifying the most-efficient tag placements, and proposing some quantitative and general guidelines useful to characterize and design this kind of new system.
\end{abstract}

Keywords: RFID; wearable; textile antenna; body-centric; on-body wireless network

\section{Introduction}

$\mathbf{R}$ adio-Frequency Identification (RFID) technology is increasingly being adopted in logistics, manufacturing, and security. Pioneering applications are currently being experimented with in sensor networks, personal healthcare, and even entertainment and social arts [1-6]. In these battery-less (say, passive) systems, the RF power required by the transponder element (the tag) to respond to the query device (the reader) is scavenged from the interrogation signal by the tag itself. Since passive RFID tags do not require regular recharging, they are suited to disposable usage, and to the pervasive and long-term distribution within environments.

In some applications, the tag device - comprising an antenna integrated with a microchip transponder, and eventually with additional sensors or actuators - needs to be worn on the human body [7-13]. Tags integrated into clothes could work as a body-centric passive RFID system, able to track people's position and/or to monitor life parameters, all the time and everywhere. If compared with active body-centric systems, RFID solutions could furthermore offer a higher degree of pervasiveness, thanks to the already available lowcost radios, the absence of rechargeable local power sources, and, not least, to the forthcoming integration of RFID functions inside mass-diffused smart phones. While active body- centric communications have been deeply addressed in the last decade [14-20], for that which concerns both the antenna design and the characterization of the propagation phenomena, passive wearable RFIDs have instead received much less attention, mainly restricted to the antenna design. These kind of systems deserve additional challenges, since RFID antennas do not transmit. Rather, the power received by the reader is backscattered from the RFID tag's antenna. Hence, the RFID antennas need to achieve efficient-energy scavenging in the presence of the very lossy human body, while keeping the antenna's size small at the involved frequency (UHF: 866$970 \mathrm{MHz}$, including European, US, and Asia subbands).

As in more-mature active body-centric systems, interesting RFID communication modalities can involve on-body and off-body links. For example, the off-body communication could be useful for locating and monitoring people inside buildings, by means of fixed readers placed in different rooms, or by a wearable reader and ambient-disseminated tags [21]. A possible application is access control in dangerous or restricted areas. The on-body communication is instead typical of unusual scenarios, where a fixed communications infrastructure is missing. This could be the case of a sportsman, a soldier, or a fireman equipped with different RFID sensors (inside a garment), interrogated by the user's handheld standard communicator in harsh environments $[22,23]$. In both 
types of link, the presence of the human body must be taken into account, in order to understand and reduce the phenomena of scattering and power absorption, and to evaluate compliance with the international regulations on electromagnetic exposure and Specific Absorption Rate (SAR) limits [24, 25].

First experimental results may be found in $[10,11]$ concerning the identification of runners in an outdoor area, also taking into account the shadowing effects in the case of formations or grouping. In [24], the power required to establish an RFID link with a human body during sleep movements of the limbs was investigated. Finally, some measurements of reading range for wearable tags placed onto the trunk and onto the arms may be found in [8], for the case of only steady postures. However, no systematic study is available to support the power-budget design for on-body and off-body RFID systems.

Starting from our recent experience with wearable RFID tags $[7,8,24]$, this paper discusses the feasibility of a bodycentric system including passive RFID textile tags, derived from a previous Teflon prototype. An articulated experimental campaign was aimed at understanding the achievable reading ranges, the minimum number of required tags, and their most suitable positions over the body for reliable on-body and offbody links. Human activity and shadowing effects were also taken into account, in order to understand the possibility of establishing robust and safe communications. Moreover, since some sensing and tracking applications are based on the processing [26] of the power backscattered by the tag toward the reader, this quantity was measured and analyzed for interesting postures and motion patterns.

Even if the results were expected to be strictly dependent on the specific environment and on the power consumption of the RFID microchip transponder, some useful normalizations were introduced, with the aim of providing first-level guidelines for the design of future passive body-centric networks.

\section{Textile Slotted-Patch Antenna}

The wearable antenna considered here for the experiments was originally proposed in [8]. It was basically a quarterwavelength patch, connected to the RFID microchip via a top $\mathrm{H}$-slot, the shape factor of which was chosen to synthesize the required complex input impedance for microchip matching. A textile version was adopted in this paper. The Teflon substrate was replaced by a $3 \mathrm{~mm}$-thick synthetic felt (Figure 1), and the conductors were made from carved adhesive copper. Due to the variability of manufacturing processes, the dielectric properties of the felt were not really assessed in the UHF band [14, 28, 29]. Hence, the electromagnetic features of our felt sample were experimentally evaluated by means of a specific parameteridentification technique, involving numerical models and measurements on planar antennas. The resulting permittivity and conductivity at the European RFID frequency of $870 \mathrm{MHz}$ were $\varepsilon=1.17$ and $\sigma=2 \times 10^{-4} \mathrm{~S} / \mathrm{m}$, to be compared with the published values of $\varepsilon=1.1$ in [29] and $\varepsilon=1.38, \tan \delta=0.0023$
$\mathrm{S} / \mathrm{m}$ in [30], both at $2.45 \mathrm{GHz}$. The antenna's matching was referred to an NXP-G2X-TSSOP-8 [31] microchip transponder, with an impedance $Z_{\text {chip }}=15-j 135 \Omega$ and a power sensitivity $P_{\text {chip }}=-15 \mathrm{dBm}$. The resulting fabricated prototype had and overall size of $7 \mathrm{~cm} \times 9 \mathrm{~cm}$ and a weight of $7.5 \mathrm{~g}$.

The electromagnetic performance of the tag has been experimentally characterized with respect to the realized gain, $\hat{G}_{T}=G_{T} \tau$, i.e., the radiation gain, $G_{T}$, of the tag's antenna placed over the body, reduced by the power-transfer coefficient $\tau \leq 1$ between the tag's antenna and the microchip. The realized gain was measured by means of the turn-on method [32], starting from the knowledge of the reader's gain, $G_{R}$; the reader-tag distance, $d$; the polarization mismatch, $\eta_{p}$, between the reader and the tag (here set to $1 / 2$ ); and finally, the turn-on power, $P_{i n}^{t o}$, e.g., the minimum input power required to be provided to the reader unit to force the remote microchip to send back its code:

$$
\hat{G}_{T}=\left(\frac{4 \pi d}{\lambda_{0}}\right)^{2} \frac{P_{\text {chip }}}{G_{R} P_{i n}^{t o} \eta_{p}} .
$$

For this purpose, a UHF long-range reader, based on the ThingMagic M5-e ASIC [33], was used. The wearable tag was interrogated by means of a $6 \mathrm{~dB}$ circularly polarized patch antenna connected to the reader. The tag was placed on the torso of a volunteer, in front of the reader's antenna, at $1.3 \mathrm{~m}$ from the ground. Ground reflections were minimized by using absorbing panels (Figure 2).

Figure 3 shows the frequency-dependent measured realized gain. Data were referred to the frontal direction, where the tag's antenna and the reader's antenna were aligned. The maximum gain at the reference frequency of $869 \mathrm{MHz}$ in Europe, ranged between $-5 \mathrm{~dB}$ and $-3 \mathrm{~dB}$, and was hence fully comparable to the Teflon prototype in $[8]\left(-4 \mathrm{~dB}<\hat{G}_{T}<-3 \mathrm{~dB}\right)$. It is worth mentioning that nearly identical results were obtained when the tag was placed onto different body segments, such as the back and the arm.

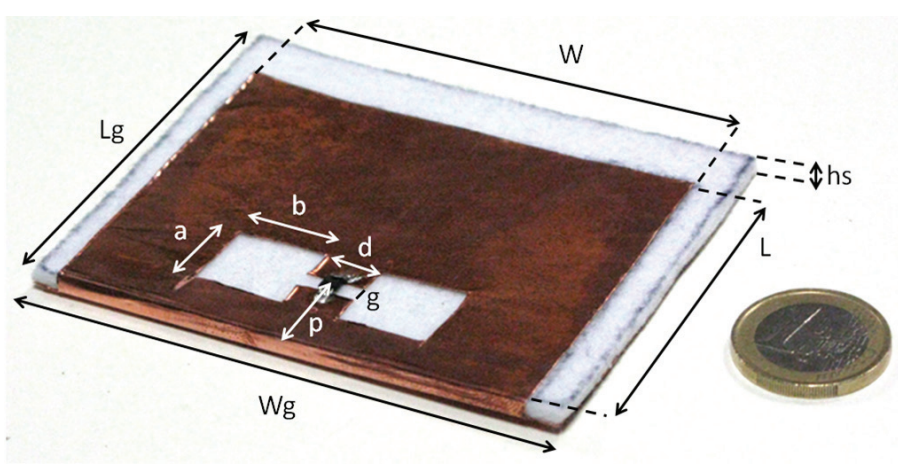

Figure 1. A prototype of a textile wearable tag, made of felt and adhesive copper. The dimensions (in $\mathbf{m m}$ ) were $L=59$, $W=80, a=15, b=15, p=14, d=10, L g=70, g=3$, $h s=3, W g=90$. 


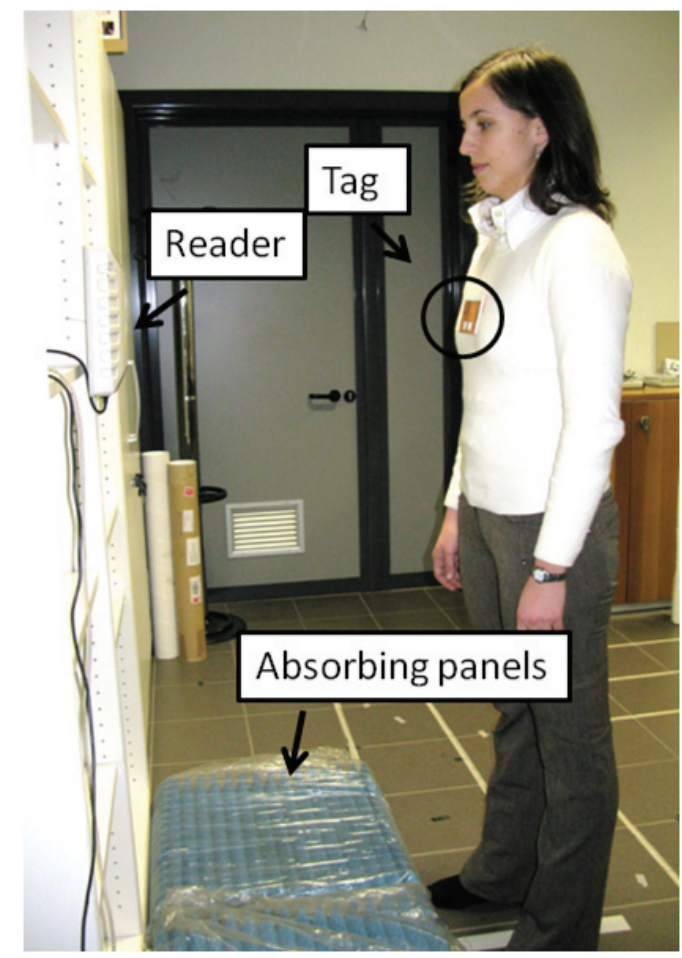

Figure 2. The setup for measuring the tag's realized gain, comprising the reader, the volunteer, and the absorbing panels. Here, the antenna was placed at the center of the human torso.

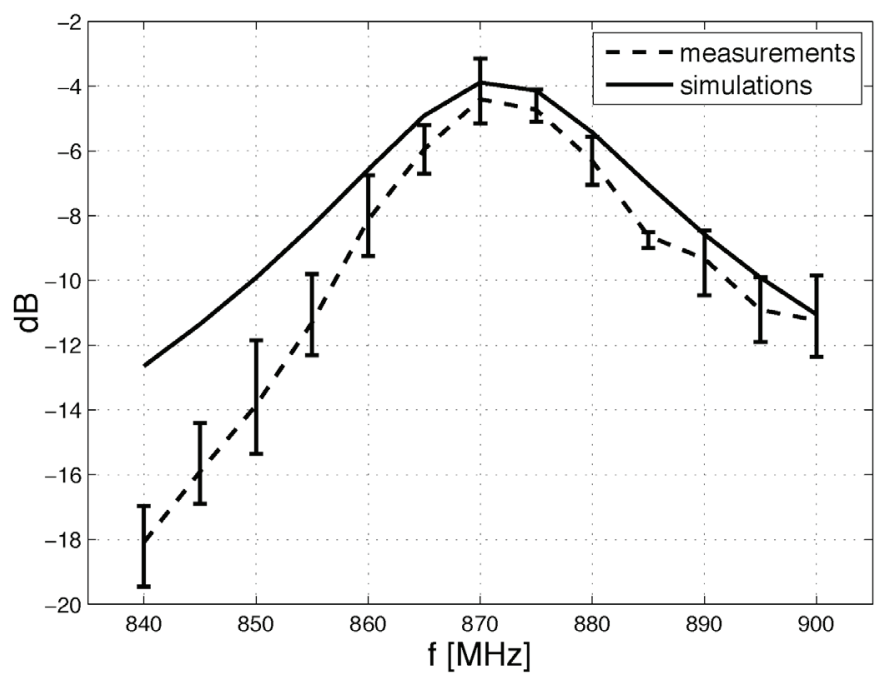

Figure 3. The maximum realized gain for the wearable tag placed on the human torso, as measured along the tag's boresight. The vertical segments give the data variability over repeated measurements.

The textile antenna was then used to characterize some passive RFID body-centric channels in both off-body and onbody links.

\section{Off-Body Communications}

In off-body communications, the most-relevant parameters to consider are the reading range of the tag in a real envi- ronment, depending on its position over the body, and the minimum number of tags and readers necessary to achieve a reliable interrogation of the subject.

The reading range of the wearable tag was measured according to the following body-centric procedure. It was definitely of interest to discriminate the region surrounding the tagged human body wherein with a reader placed any way, at least a tag was successfully interrogated. It was expected that such a region would depend on the position of the tag over the body and on the reader-emitted power, as well as on the reader antenna's pattern. As for the previous Teflon prototype in [8], the textile tag also revealed very little sensitivity to the body's position in terms of impedance matching and maximum gain, thanks to the presence of the ground plane. Nevertheless, the shape of the reading region may be dependent on such a position, due to the specific shadowing caused by body segments themselves, and by absorption modality. Hence, to discuss this issue, three different tag placements were considered (front torso, arm, and back), each in horizontal and vertical orientation, as shown in Figure 6.

For the sake of simplicity, the reading region was measured in a reciprocal way, e.g., the reader was fixed in some location in the room, while the tagged person walked away from the reader along the boresight of the reader's antenna (see Figure 4). The actual reader-body distance was considered to belong to the reading region of the tag if the tag was able to correctly reply to the reader's interrogation. The procedure was repeated for rotations of the body of $\left\{0^{\circ}, 90^{\circ}, 180^{\circ}, 270^{\circ}\right\}$ degrees with respect to the reader's antenna.

The measurements were performed in a $5.5 \mathrm{~m} \times 5.5 \mathrm{~m}$ $\times 3 \mathrm{~m}$ office room, the inner furniture having been removed. The same reader's antenna and long-range reader as before were placed on the middle of a side wall, $1.3 \mathrm{~m}$ from the floor. The reader emitted a fixed power of $3.2 \mathrm{~W}$ EIRP, which is the maximum value allowed by the European regulations. The space sampling rate was $30 \mathrm{~cm}$.

In general, the reading range in real environments was affected by the presence of walls and furniture in a way that was also dependent on the reader's power and the microchip's sensitivity. All of these issues were extensively addressed in [21], but it is useful to recall that in the case of low-gain tags - as for the wearable textile patch - the effect of the side walls of the room considered here could be neglected. Hence, the results in the following sections may be extended to different environments by introducing the two-ray correction terms as in [21].

An example of a measurement is given in Figure 5, where the solid dots indicate a successful interrogation of the tag when the person stood at that point. The empty circles instead represent a reading failure. By considering that the transition between the reading region and the unreachable region was not sharp due to the formation of diffraction fringes [21], the following metrics are assumed to define the maximum reading distance, $d_{\max }\left(\phi_{n}\right)$, in a given direction $\phi=\phi_{n}$. The distance $d_{\text {max }}\left(\phi_{n}\right)$ was calculated from the human body to the first point 


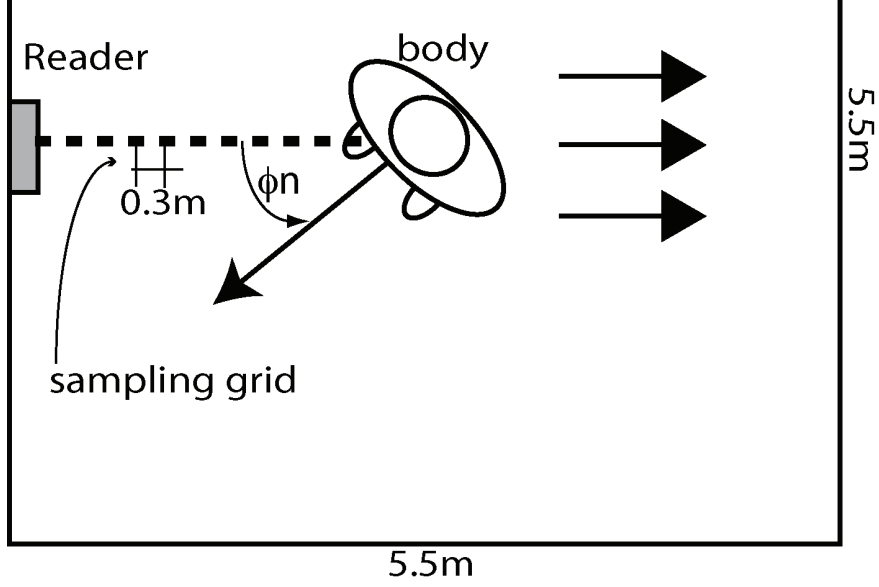

Figure 4. The reader-tag mutual orientations during measurements to evaluate the extension of the body-centric reading region. The volunteer equipped with a wearable tag walked away from the reader's antenna, which was placed on the middle of a wall inside a $5.5 \mathrm{~m} \times 5.5 \mathrm{~m} \times 3 \mathrm{~m}$ room, at a distance of $1.3 \mathrm{~m}$ from the floor. The tag was interrogated when the volunteer was standing at the dotted positions, in $30 \mathrm{~cm}$ steps. Each point was said to be accessible by the RFID system if the tag was able to answer the reader. The experiment was repeated for four orientations of the body with respect to the reader's antenna, e.g., for $\varphi_{n}=\left\{0^{\circ}, 90^{\circ}, 180^{\circ}, 270^{\circ}\right\}$, to collect the two-dimensional reading range. of faulty interrogation (empty circle) followed by less than two consecutive points of successful interrogation (filled circles). In other words, we gave a positive value to even the very isolated failure points, in consideration that the subject could be moving and so the subject could still be statistically detected with overall continuity.

The results of the measurement campaign are presented in a compact form in Figure 6. The overall maximum reading distance was about $5 \mathrm{~m}$, arising in Case $\mathrm{E}$ (with the tag over the arms with horizontal orientation). In most of the configurations considered, the maximum distance was about $4.5 \mathrm{~m}$, confirming that the maximum gain of the antenna had very little sensitivity to the position over the body. The reading distances out of the antenna's boresight were instead dependent on the dielectric losses of the human body, which produced significant absorption and shadowing, especially in the case of tags placed on the shoulder. The polarization of the tag, e.g., the orientation of the radiating edge of the patch, seemed to play a significant role in the achievable reading ranges, even if the physical reasons were not immediately clear. Vertical polarization performed far better in the case of placement over the chest, while horizontal polarization appeared more suitable for placement over shoulders and arms.

However, in none of the considered tag placements was it possible to achieve a nearly uniform coverage with just a single tag. However, by a combined use of tags it was expected to achieve a reliable RFID link, for instance, by placing one tag over the torso and the other over the back $(\mathrm{A}+\mathrm{H})$, or instead, a single tag for each arm (F(right) $+\mathrm{F}($ left $))$. The combined results are shown in Figure 7, where nearly circular body-centric interrogation regions were visible. These diagrams represented the area in which wherever a reader emitting $3.2 \mathrm{~W}$ EIRP is

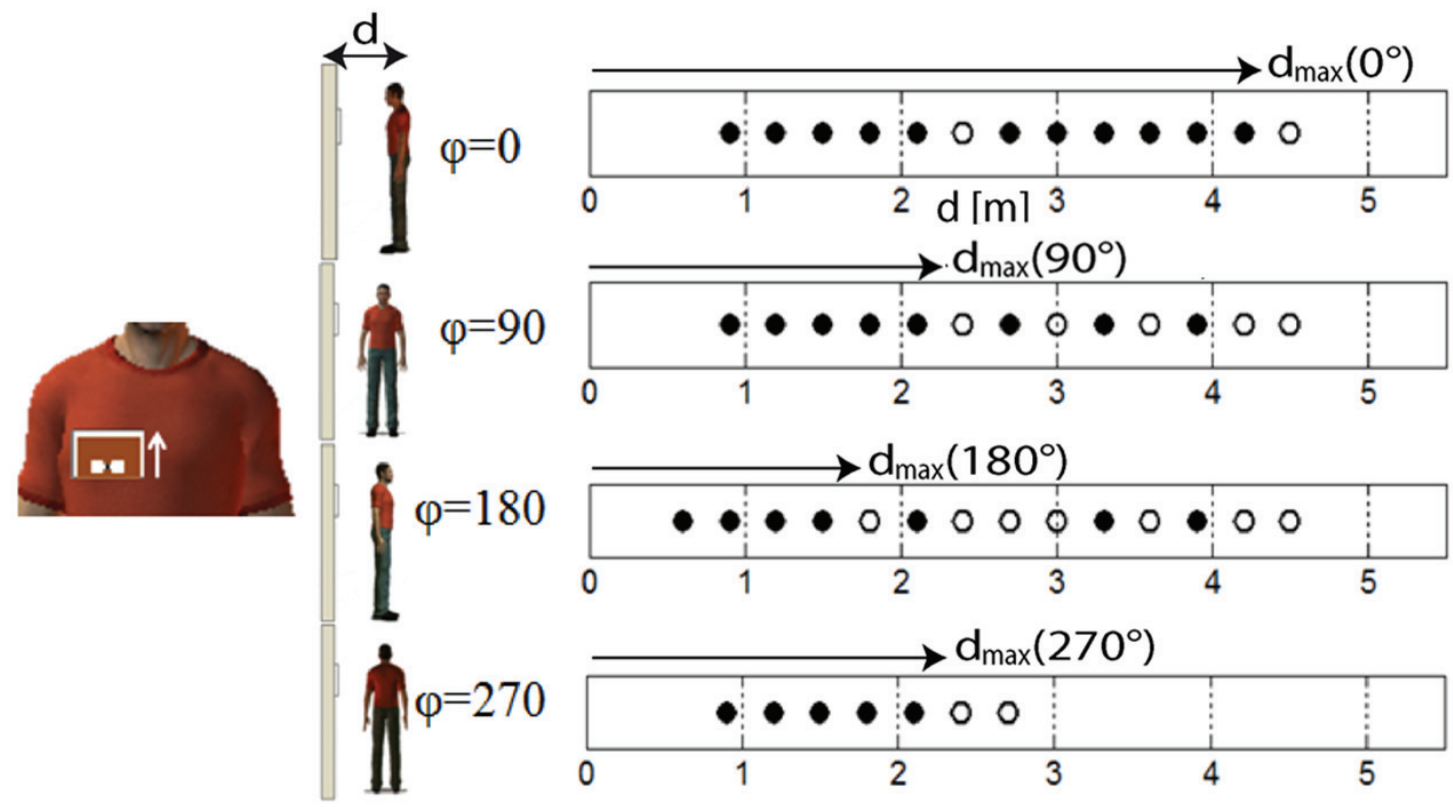

Figure 5. An example of the reading-region measurements when the tag was placed over the torso with vertical polarization. For each reader-body orientation, a filled circle indicates a successful reading of the tag at that point, while an empty circle indicates a reading failure. The maximum reading distance, $d_{\text {max }}$, along each direction was calculated from the human body to the first point of fault interrogation followed by less than two consecutive points of successful interrogation. 


\begin{tabular}{|c|c|c|c|c|}
\hline Position & $\underbrace{q_{d m a x}^{R}}_{\phi=0^{\circ}}$ & $\vartheta^{R}$ & \begin{tabular}{c}
$\bigvee_{\phi=180^{\circ}}^{R}$ \\
\hdashline
\end{tabular} & $\bigoplus_{\phi=270^{\circ}}^{p}$ \\
\hline A & 4.2 & 2.1 & 1.5 & 2.1 \\
\hline B & 1.8 & 3.3 & 0.6 & 1.5 \\
\hline$c$ & 2.1 & 2.1 & 2.1 & 1.5 \\
\hline D & 4.2 & 3.6 & 1.2 & 0.6 \\
\hline$E$ & 5.1 & 2.7 & 2.4 & 0.6 \\
\hline$F$ & 4.5 & 4.5 & 3.3 & - \\
\hline G & - & 4.5 & 4.8 & 2.1 \\
\hline$H$ & - & 4.5 & 4.5 & 3.6 \\
\hline
\end{tabular}

Figure 6. The reading distances (in meters) measured at four different angles from the thorax's normal axis, for several positions and orientations of the body-worn tag.

placed in front of the person, it was possible to monitor the subject independently of the subject's position and orientation. Even better results may be achieved by using three tags: two of them over both of the arms, and one over the chest.

In conclusion, a single reader permits establishing a reliable RFID link with a person equipped with two or three tags within a room of size $4 \mathrm{~m}$ by $4 \mathrm{~m}$. Four readers, each placed on each side wall, would instead enable continuous interrogation within a room that was four times larger $(9 \mathrm{~m}$ by $9 \mathrm{~m})$.

The above results were obtained for a particular choice of the reader's power and of the family of microchips. However, such data can also be useful in case of different choices of power parameters by introducing the effective microchip's sensitivity [21], an aggregate performance indicator for the tag, defined as

$$
p_{\text {chip }}=\frac{P_{\text {chip }}}{\hat{G}_{T}} .
$$

This gives the minimum radio-frequency power that the tag has to collect to exhibit the same averaged free-space reading distance as a perfectly matched tag placed over a lossless object (averaged $\hat{G}_{T}=1$ ). In our particular case, $p_{\text {chip }, 0}=90 \mu \mathrm{W}$. From Equation (1), the maximum reading distance is linearly dependent on the ratio $\sqrt{\operatorname{EIRP} \hat{G}_{T} / P_{\text {chip }}}$, where $\operatorname{EIRP}=G_{R} P_{\text {in }}$ . Therefore, for a different choice of power and effective microchip sensitivity, an estimation of the reading range, $d_{\text {max }}\left(\frac{E I R P}{P_{\text {chip }}}\right)$, may be roughly deduced from the values of the reading distances given in Figure 6 (now denoted as $d_{\max , 0}$ for $E I R P_{0}=3.2 \mathrm{~W}$ ) by means of the following scaling formula:
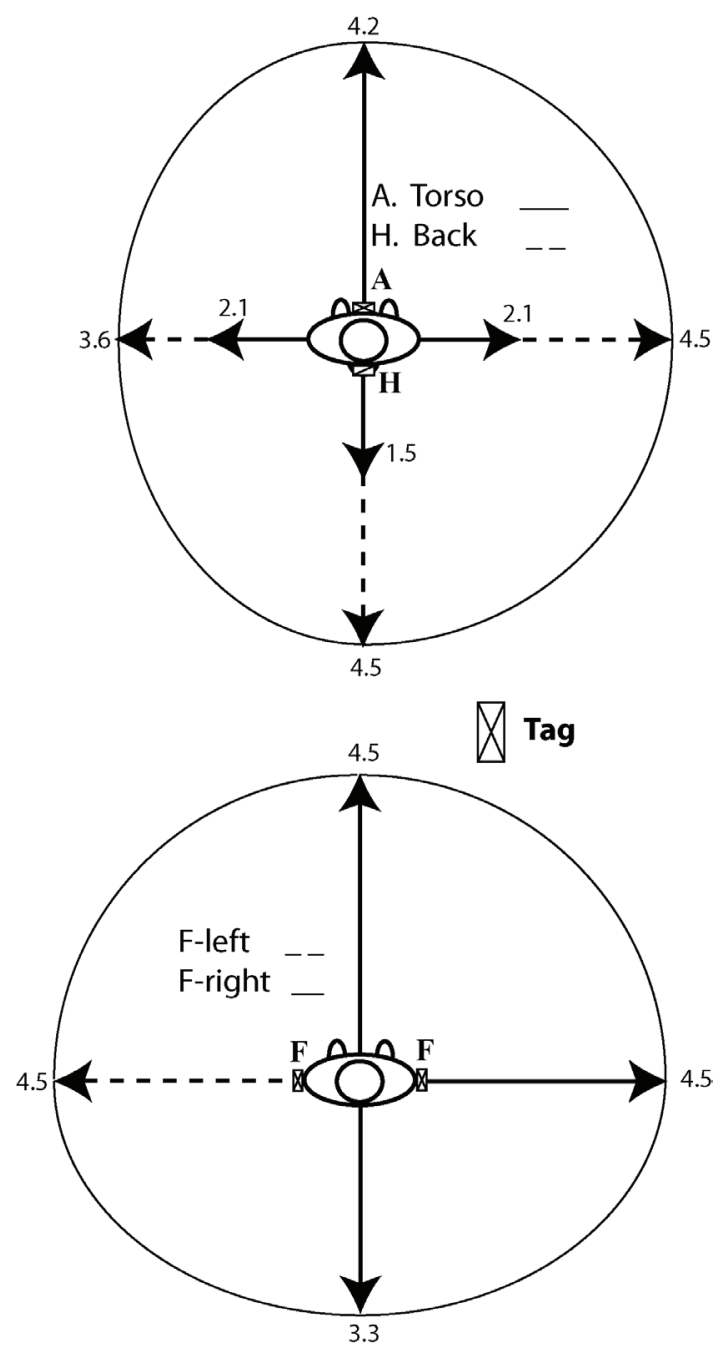

Figure 7. The body-centric reading regions for two couplets of tags placed on the human body: (top) tags over the front torso and shoulders (Cases $\mathrm{A}$ and $\mathrm{H}$ in Figure 6); (bottom) tags over both of the arms (Case $F$ in Figure 6). The continuous and dashed arrows indicate the maximum reading distances, $d_{\max }\left(\phi_{n}\right)$, of the two tags in the specific direction, and the ellipse-like region gives the estimate of the overall resulting body-centric reading region for the combined two-tag system. 


$$
\begin{aligned}
d_{\max }\left(\frac{E I R P}{P_{\text {chip }}}\right) & =\sqrt{\frac{P_{\text {chip }, 0}}{E I R P_{0}}} \sqrt{\frac{\text { EIRP }}{P_{\text {chip }}}} d_{\text {max }, 0} \\
& =5.3 \times 10^{-3} \sqrt{\frac{E I R P}{P_{\text {chip }}}} d_{\text {max }, 0} .
\end{aligned}
$$

\section{On-Body Communications}

In case of on-body communications, in addition to the tag placements it is of interest to also analyze the effects of body posture and activity on link shadowing. Depending on the reader-tag position, different propagation phenomena are excited [34]. Creeping waves can be predominant for communications between adjacent body segments (waist-torso, armforearm), while diffracted and reflected free-space waves could be considered responsible for the communications between distant regions, such as arm-leg, head-waist, etc. In both cases, it is not appropriate to consider the typical far-field approximation. The quality of the established links can hence be characterized according to the turn-on power, $P_{i n}^{t o}$, enabling the activation of the tag in the specific position, and according to the percentage of successfully interrogations. In order to provide a general performance indicator such as Equation (2), the turn-on power is here normalized by the microchip's effective sensitivity, $P_{\text {chip }}$ :

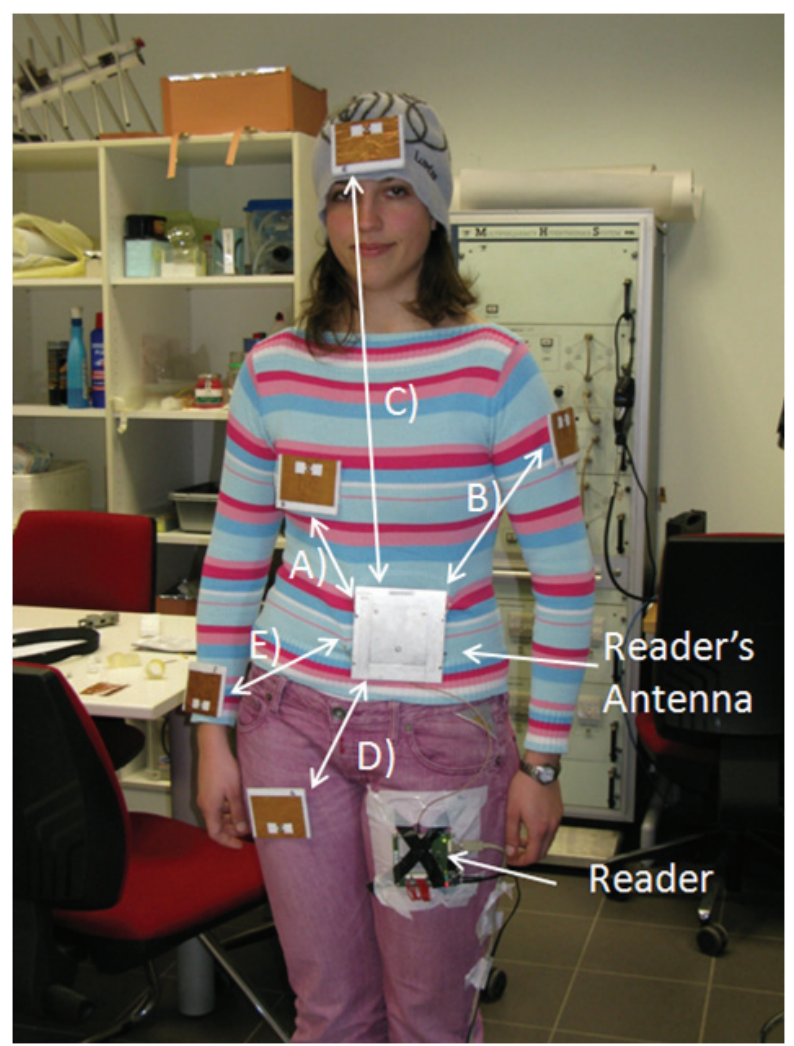

Figure 8. The antenna positions on the body. Five different links were considered here. The reader was placed at the waist, slightly on the left.
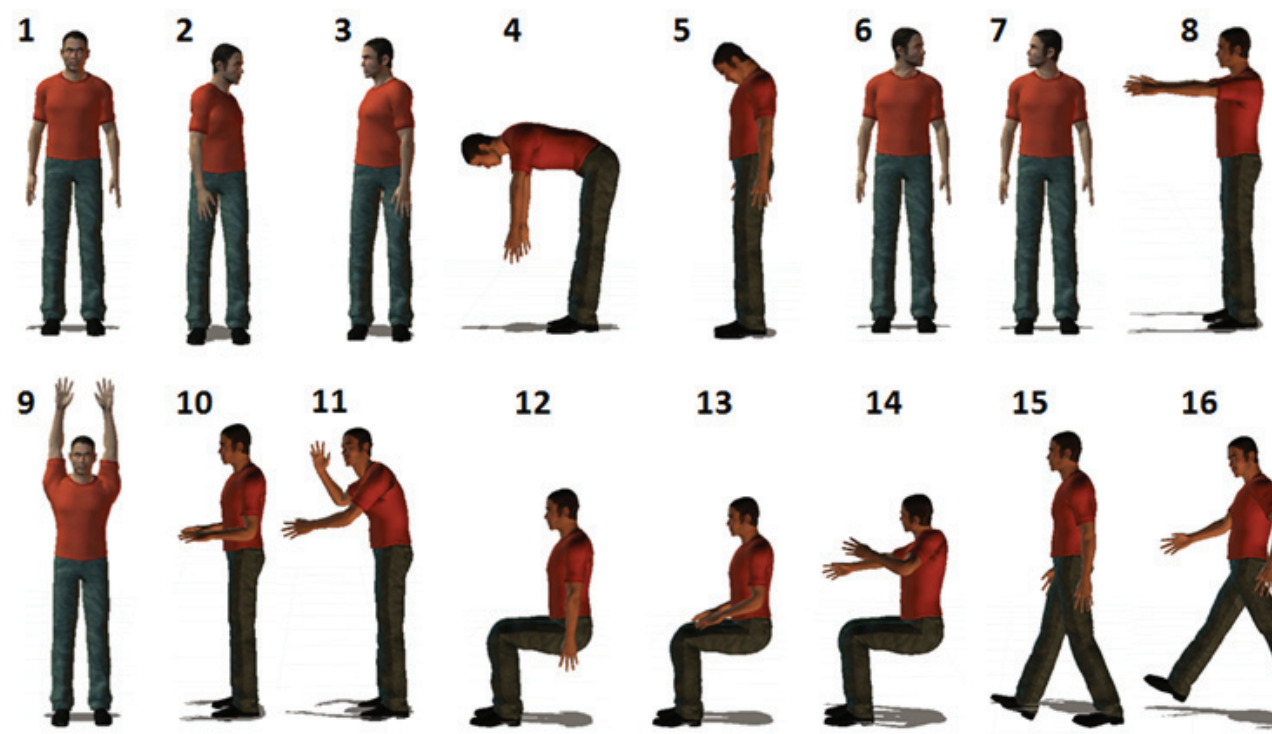

13

14

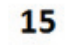

16
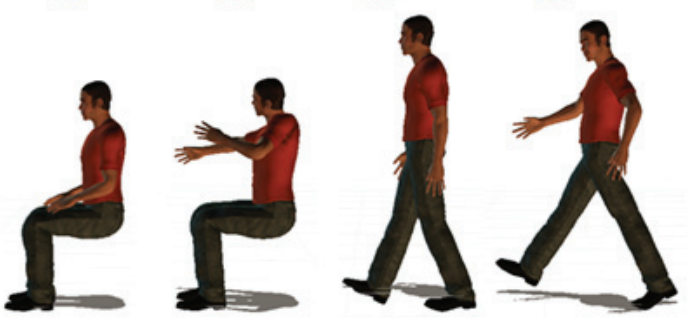

17

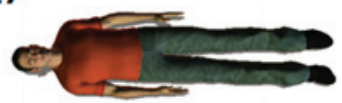

19

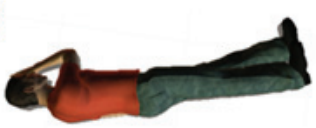

18

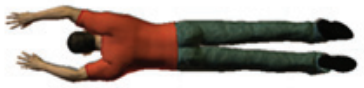

20

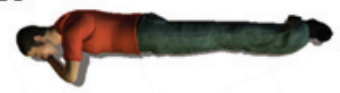

Figure 9. The postures assumed by the subject during the measurements. In the first set of fourteen configurations, the subject stood or sat according to typical human activities. Two postures (15 and 16) were in motion. In the last set of four postures, the subjects laid on the floor, simulating healthy and pathological conditions, such as the later safety position (LSP). 
Table 1. The normalized turn-on powers, $p_{t o}$, for the postures in Figure 9 and the on-body RFID links in Figure 8.

\begin{tabular}{|c|c|c|c|c|c|}
\hline \multirow{2}{*}{ Posture } & \multicolumn{5}{|c|}{ Normalized Turn-On Power } \\
\cline { 2 - 6 } & Link A & Link B & Link C & Link D & Link E \\
\hline \hline 1 & 0.08 & 0.44 & 0.56 & 0.39 & 0.62 \\
\hline 2 & 0.05 & 1.76 & 0.55 & 0.62 & 1.4 \\
\hline 3 & 0.09 & 2.79 & 1.76 & 0.5 & 1.4 \\
\hline 4 & 0.04 & 3.51 & 1.11 & 0.35 & 1.76 \\
\hline 5 & 0.1 & 0.35 & 0.35 & 0.62 & 0.62 \\
\hline 6 & 0.09 & 0.7 & 0.88 & 0.62 & 0.62 \\
\hline 7 & 0.08 & 0.35 & 1.11 & 0.39 & 0.79 \\
\hline 8 & 0.07 & 1.4 & 0.7 & 0.62 & 4.42 \\
\hline 9 & 0.09 & 4.96 & 0.44 & 0.7 & - \\
\hline 10 & 0.05 & 0.99 & 0.56 & 0.99 & 0.5 \\
\hline 11 & 0.05 & 0.57 & 0.44 & 0.39 & 0.39 \\
\hline 12 & 0.04 & 0.4 & 0.7 & 0.35 & - \\
\hline 13 & 0.04 & 0.35 & 0.88 & 0.62 & 0.39 \\
\hline 14 & 0.05 & 0.62 & 0.25 & 0.25 & 0.44 \\
\hline 15 & 0.05 & 0.5 & 0.39 & 0.44 & 0.49 \\
\hline 16 & 0.05 & 0.35 & 0.44 & 0.28 & 0.44 \\
\hline 17 & 0.08 & 1.25 & 0.7 & 0.41 & 8.23 \\
\hline 18 & 5.57 & - & 9.24 & - & - \\
\hline 19 & 0.08 & 6.54 & 0.88 & 0.33 & 1.25 \\
\hline 20 & 0.1 & 0.92 & 1.11 & 0.79 & - \\
\hline & & & & & \\
\hline
\end{tabular}

$$
p_{t o}=10^{-3} \frac{P_{\text {in }}^{\text {to }}}{P_{\text {chip }}}
$$

Such a parameter could hence be considered as a kind of "transfer function" of the system: the lower $p_{t o}$, the more efficient and reliable is the link.

In this second measurement campaign, the reader's antenna was a smaller, linearly polarized, quarter-lambda patch (PIFA), with a maximum $3.3 \mathrm{~dB}$ gain, that was suited for placement onto the volunteer's body, close to the waist. The reader unit was also attached onto the body (Figure 8).

The PIFA interrogated five wearable tags, attached onto the torso, arm, head, leg, and wrist, respectively. To improve polarization matching, the PIFA was oriented so that its polarization vector was always parallel to that of the tags in all the experiments. During the measurements, the volunteer took twenty different "static" (1-14) and "moving" (15-16) postures, illustrated in Figure 9. The last four positions corresponded to the subject lying on the ground and could be representative of particularly dangerous situations, such as fainting or accidents.

The measurement results are reported in Table 1. In our specific case, by considering the same microchip sensitivity as before, the turn-on power ranged between $8 \mathrm{dBm}$, corresponding to $p_{t o}=0.07$ for Link A, and $29 \mathrm{dBm}\left(p_{t o}=9.24\right)$, for Link C. As expected, thanks to the smallest and stable distance, the most-efficient link was that involving the tag over the front torso (Link A). Independently of the different postures and movements, Link A required the minimum activation power, and it was only a little sensitive to the shadowing effects of the body. Link E (waist-wrist) was instead the most sensitive to the mismatch polarization and to the shadowing produced by body movements: in Postures 9 and 12, the tag placed on the wrist resulted in being even unreadable. Among the prone positions, recumbent Posture 18 was the most challenging position in which to establish an RFID link, due to the close presence of the ground within the reader-tag link. In this case, the required turnon power was two orders of magnitude higher than in case of the standing positions. It is moreover worth observing that the two side-lying postures (19 and 20) yielded completely different turn-on powers, due to the asymmetric position of the reader/ tags over the body, and due to the combined shadowing effects of floor and arms.

Some statistics are presented, having performed $N_{T}$ interrogations of Tags $\mathrm{A}$ and $\mathrm{D}$ in the 20 postures with a fixed reader power of $P_{i n}=20 \mathrm{dBm}$, such that at least the Links A, D were fully active. 
If the reader received $N_{R}$ responses from the tag, a quality parameter was the percentage of answers, $\alpha$, defined as

$$
\alpha=100 \frac{N_{T}}{N_{R}} .
$$

The reader was set to perform nine polls per second, each interrogation period was $10 \mathrm{~s}$, and hence $N_{T}=90$. Both vertical and horizontal tag orientations were considered, and, as above, the PIFA was properly rotated to preserve the polarization matching. The results are shown in Figure 10 and Figure 11 for the vertical and horizontal orientations of the antennas.

Link A was rather robust, with a percentage of correct answers exceeding $70 \%$ in almost every case. Link D was instead much more sensitive to the human's activity, with a lower percentage of answers, sometimes close to zero. The mutual orientation between the reader's antenna and the wearable tags affected the reliability of the links. It was apparent that Link A exhibited the best performance in the case of the verticalpolarization setup, since both the reader's antenna and the tag were oriented along the directions of maximum radiation, e.g., the radiating edges of the two patches were facing each other. For the same effect as before, Link D instead appeared to be the better performing link in the case of horizontal polarization.

In any case, the lying positions were the most reliable, due to the high variability of the body segments' positions. Except for the supine posture, the statistics of the readings could be very unstable in the other configurations, regardless of the required activation power. In these cases, the torso-waist channel results were also the most efficient.

\section{Measurement of Backscattered Power}

The on-body configurations $\mathrm{A}, \mathrm{D}$, and $\mathrm{E}$ are here also characterized in terms of the backscattered power, $P_{B S}$, received by the reader. This quantity is in general less critical than the turn-on power in view of estimating the quality of the RFID link, since the communication bottleneck is the forward link. Nevertheless, there are some emerging applications wherein the variation of the backscattered power is correlated to some physical property of the tag, and, in turn, to the change of the tagged object or of the nearby environment, with the purpose of achieving a sort of RFID passive sensing. This is the case when the tag is coupled to a chemically sensitive substrate detecting the presence of gases, or when the antenna itself is deformed by motion [35].

For this purpose, three rather common postures of onbody communication were considered, with the aim of analyzing the expected fluctuations of the received signals and an eventual correlation with the periodicity of motion. In the first posture, the subject stood up motionless, as in Figure 9. In the second posture, the person instead walked along a straight path, inside a corridor, with controlled steps: first a slow walk $(60 \mathrm{~cm} / \mathrm{s}$ per second) and then a faster motion $(120 \mathrm{~cm} / \mathrm{s})$. In the third posture, the subject folded his or her arms up and down, as shown in the inset to Figure 14. The same reader and PIFA antenna as before were used in the three experiments. The input power to the reader's antenna was fixed at $P_{i n}=20 \mathrm{dBm}$. The backscattered power was deduced from the RSSI (receivedsignal strength indicator) provided by the reader, by means of the following conversion equation (specific for that reader):

$$
P_{B S}=0.8 R S S I+-G_{L N A}-96-0.8 R S S I_{t h},
$$

with $R_{S S I} I_{t h}=48$, and $G_{L N A}$ being the gain of the low-noise signal amplifier, defined in the communication register. For generality, the backscattered power was normalized by the input power:

$$
p_{B S}=\frac{P_{B S}}{P_{i n}} .
$$

Figure 12 shows that the fluctuation of $p_{B S}$ for both the links were comparable, and were of the order of $\pm 1 \mathrm{~dB}$ around the average value. This uncertainty needs to be carefully taken into account in estimating the dynamic range of any sensing application based on RSSI processing.

The measurements in for the walking case (Figure 13) showed that the motion affected the two links in a different way. While Link A (with the tag on the chest) remained approximately oscillating around the same average value, but with higher fluctuations ( $\pm 2 \mathrm{~dB}$ ), the backscattered power for Link D (with the tag on the leg) presented a much higher variability, due to the time-changing mutual position between the leg and the reader's antenna. In this case, the amplitude of the fluctuations was $\pm 4 \mathrm{~dB}$, and they followed the walking rhythm. The period in the first part of the trace was approximately $2 \mathrm{~s}$, corresponding to a complete movement of the tagged leg at $60 \mathrm{~cm} / \mathrm{s}$, and which then changed to $1 \mathrm{~s}$ in the second trace, when the walker's speed doubled.

In the same way, Link E was characterized during periodic movements of the arm. Figure 14 shows the fluctuation of the $p_{B S}$ in the case of a $2 \mathrm{~s}$ motion periodicity. When the arm was outstretched (parallel to the body), the reader correctly detected the backscattered power. In the case where the arm was folded (orthogonal to the reader), the microchip did not receive enough power to activate, and no signal was detected at the reader's side. Hence, a cluster-like response was observed, with the clear possibility of recognizing the periodicity of the motion.

From these results, it was apparent that the use of backscattered power to retrieve the output of an RFID sensor looked very challenging for links involving moving limbs, since the theoretical dynamic range of the sensing mechanism had to be much larger than $8 \mathrm{~dB}$ to be recognized by the reader. Nevertheless, it was evident that the RSSI may be used as an indicator of motion of a specific part of the body. This could be attractive in itself for collecting statistics about repeated movements of working people, and for remote healthcare of elders. 
Tag response on chest
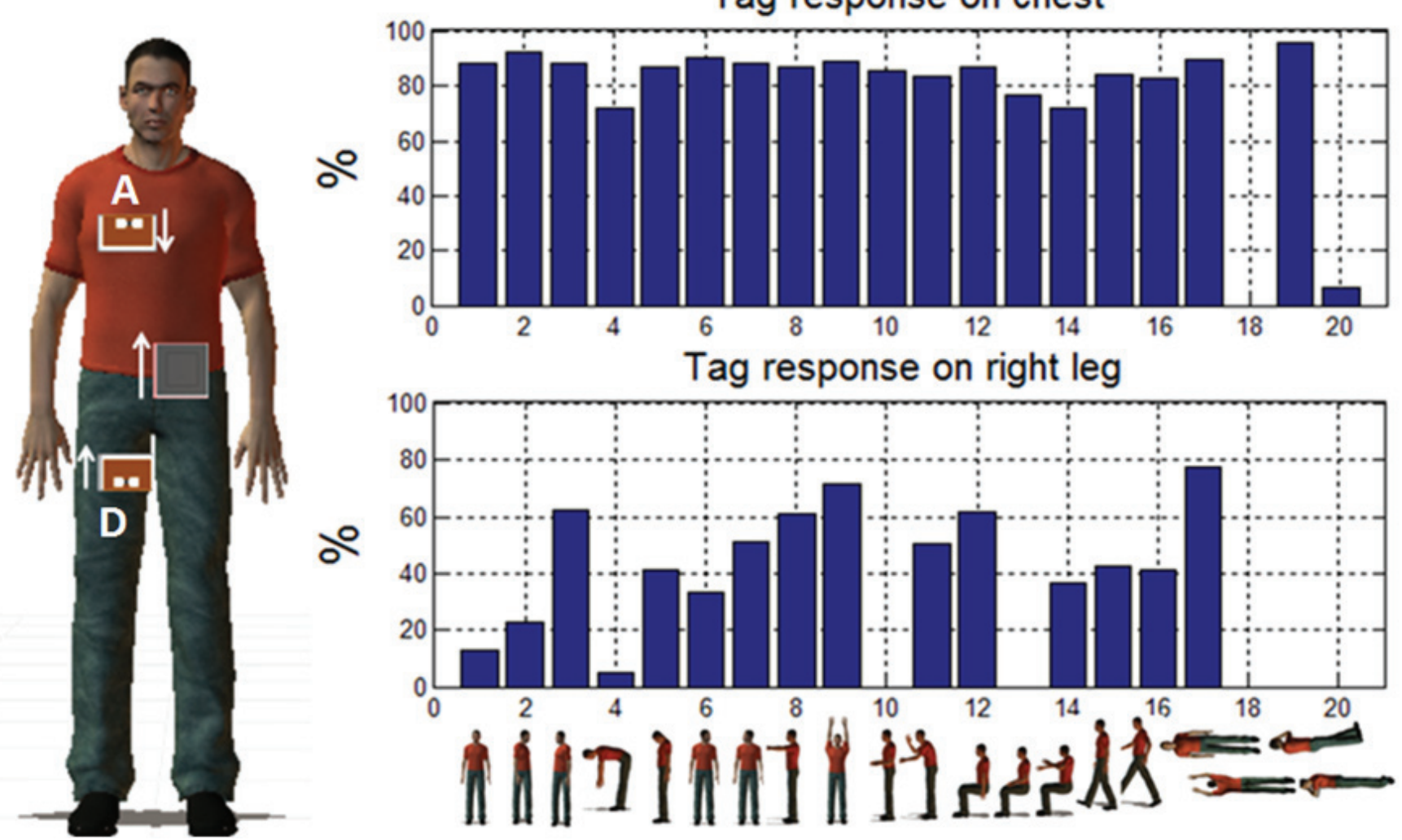

Figure 10. The channel reliability, $\alpha$, for several postures, expressed in terms of the percentage of answers with the antennas in vertical polarization.

Tag response on chest
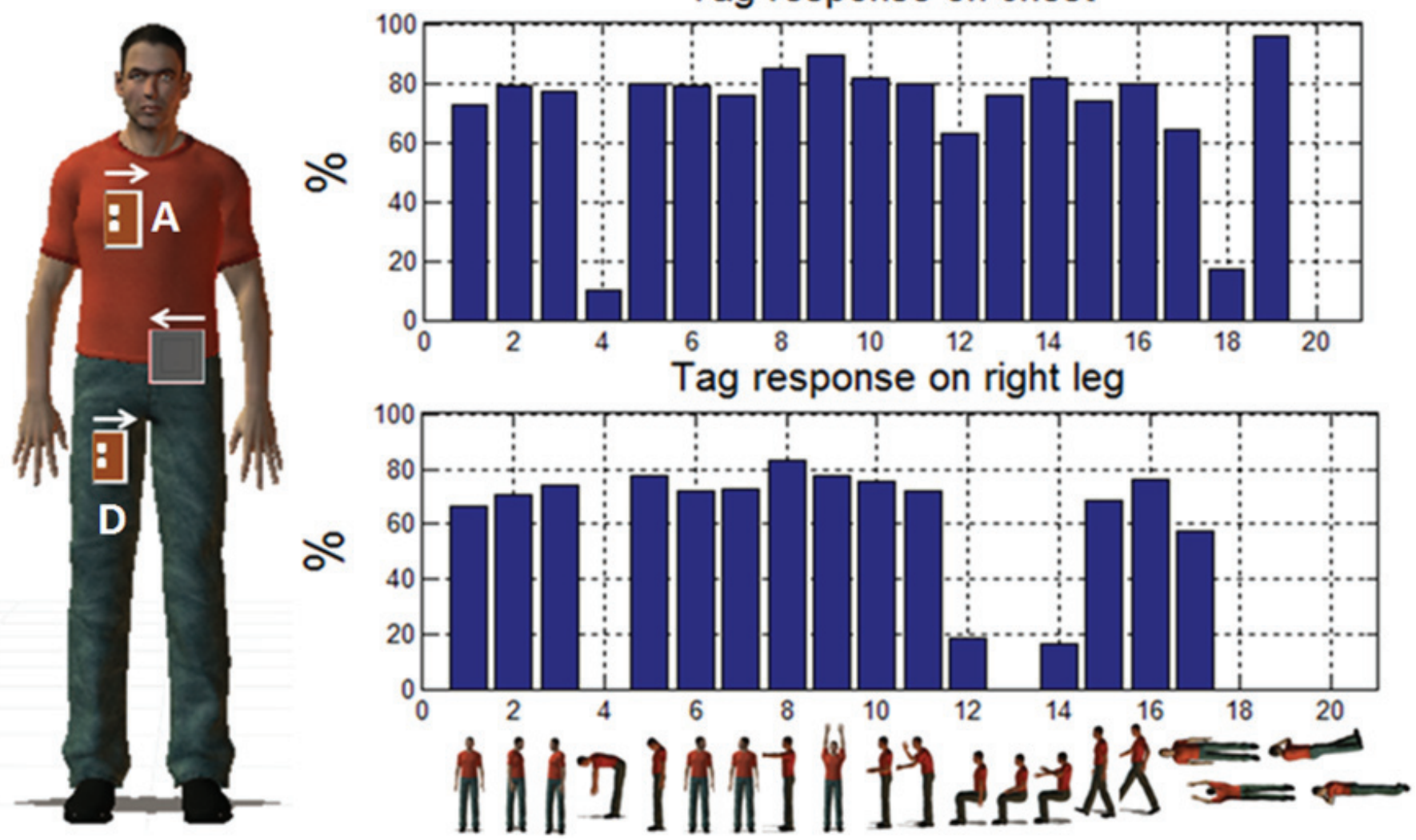

Figure 11. As in Figure 10, but with the antennas in the horizontal orientation. 


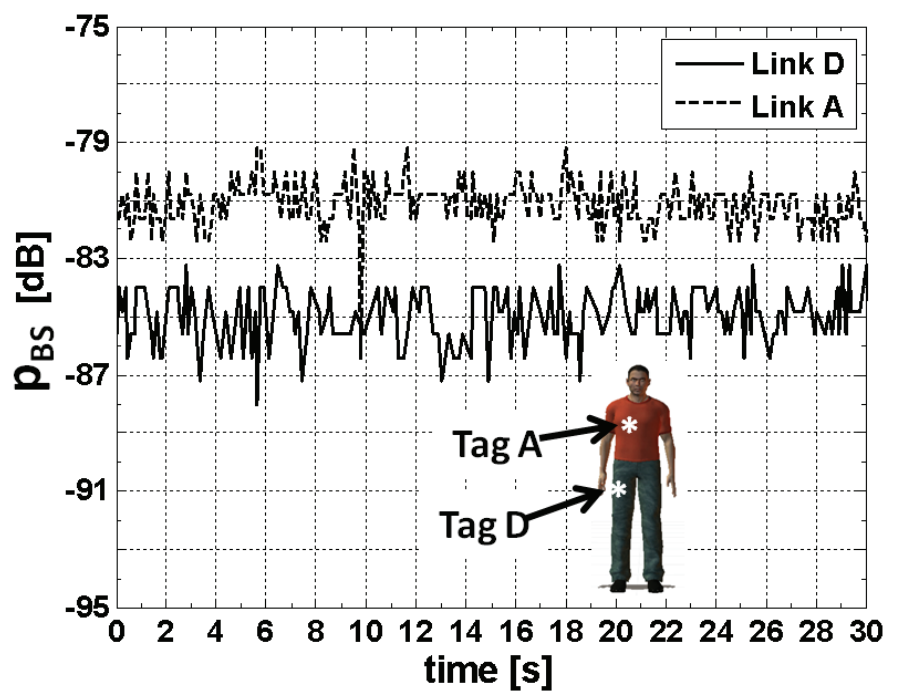

Figure 12. The normalized backscattered power, $p_{B S}$, from Tags $A$ and $D$, as collected by the on-body PIFA antenna placed as in Figure 10, when the subject was standing motionless.

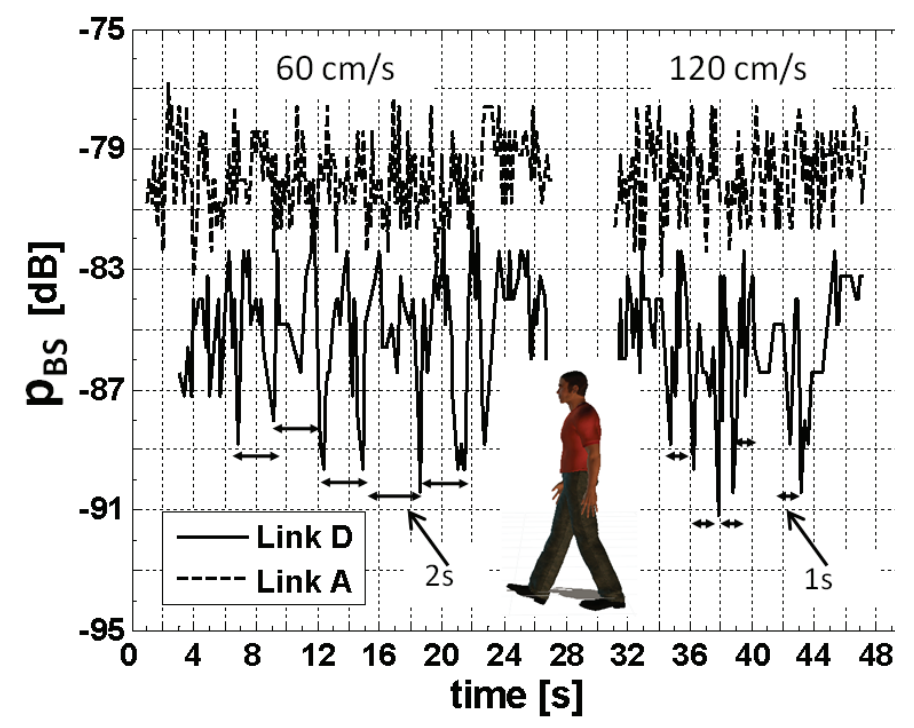

Figure 13. The normalized backscattered power, $p_{B S}$, from Tags $A$ and $D$, when the person walked along a straight line within a corridor, initially with a velocity of $60 \mathrm{~cm} / \mathrm{s}$, and then at $120 \mathrm{~cm} / \mathrm{s}$. The $p_{B S}$ power looked to be able to follow the walking dynamics.

\section{Safety Issues}

In order to discuss the compliance of the wearable system with exposure limits, some issues concerning the Specific Absorption Rate (SAR) and the radiated electromagnetic field are discussed here for the on-body and the off-body setups with the help of numerical electromagnetic simulations. In the first case, the same FDTD torso model previously adopted for the design of the wearable antenna was applied to estimate the resulting SAR distribution at $870 \mathrm{MHz}$, averaged over $10 \mathrm{~g}$ of tissue. The reader's PIFA antenna was placed on the middle of the external surface of the cylinder, and radiated a fixed $0.5 \mathrm{~W}$ power, as in the on-body experiments. The diagrams in Fig- ure 15 for both vertical and horizontal placements of the PIFA had to be compared with the considered SAR limit of $2 \mathrm{~W} / \mathrm{Kg}$ [36]. As expected, the maximum SAR occurred underneath the antenna, in correspondence with the muscular tissue, but it was one order of magnitude smaller than the absorption limit. This means that the cohabitation of the reader's PIFA with the body was safe, even for the case of the maximum emitted power allowed by European (3.2 W EIRP) and US (4.0 W EIRP) regulations.

Concerning off-body communication, the experimental reading distance was simply discussed with respect to the maximum limit, $E_{0}$, imposed on the field radiated by the reader. The study in [21] addressed this topic by introducing a forbidden region all around the reader, wherein the emitted field strength exceeded the allowed limit, and hence no person should be present inside for more than a given time, specific to the local regulation. In particular, the simulations in [21] showed that even in the case where the reader radiated $3.2 \mathrm{~W}$ EIRP, the extension of the forbidden region was less than $50 \mathrm{~cm}$ for $E_{0} \leq 20 \mathrm{~V} / \mathrm{m}$. Moreover, such a distance was practically further reduced for the case of duty cycles $d<1$. For instance, assuming a typical inventory communication between reader and tag with ten interrogations per second, the resulting forbidden distance would be halved with respect to the case of continuous interrogation. Therefore, the off-body link was fully compatible with local regulations for body-tag distances larger than a small fraction of a meter.

\section{Conclusion}

The experiments presented demonstrated that passive body-centric RFID links are feasible within a regular indoor room with the technology available today, and within safety regulations. Actually, the on-body link may be established using a query power of the order of just $10 \mathrm{dBm}$ in case where the tag is placed over the torso and the reader's antenna is on the waist. This power requirement is compatible with pico-

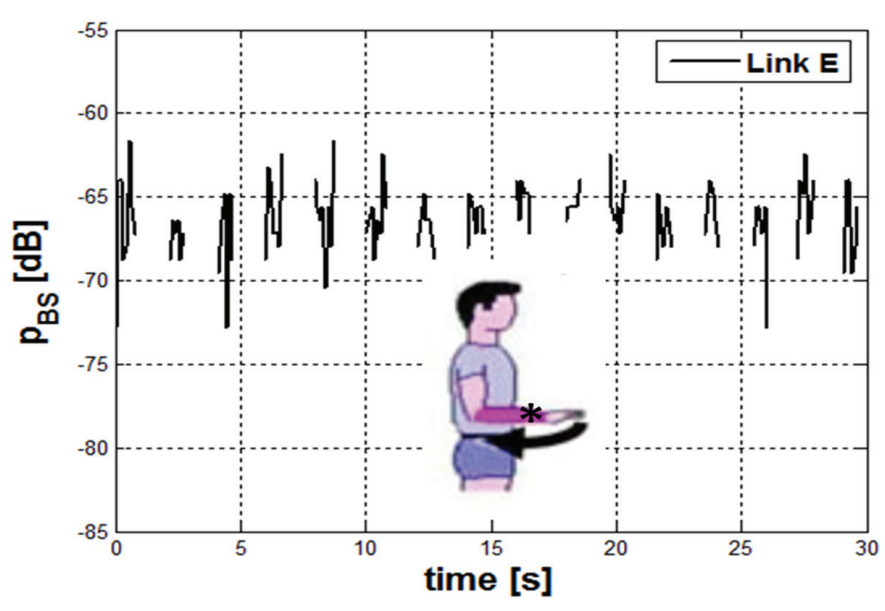

Figure 14. The normalized backscattered power, $p_{B S}$, from Tag $E$ when the subject's arm was moving up and down at a frequency of $0.5 \mathrm{~Hz}$. 


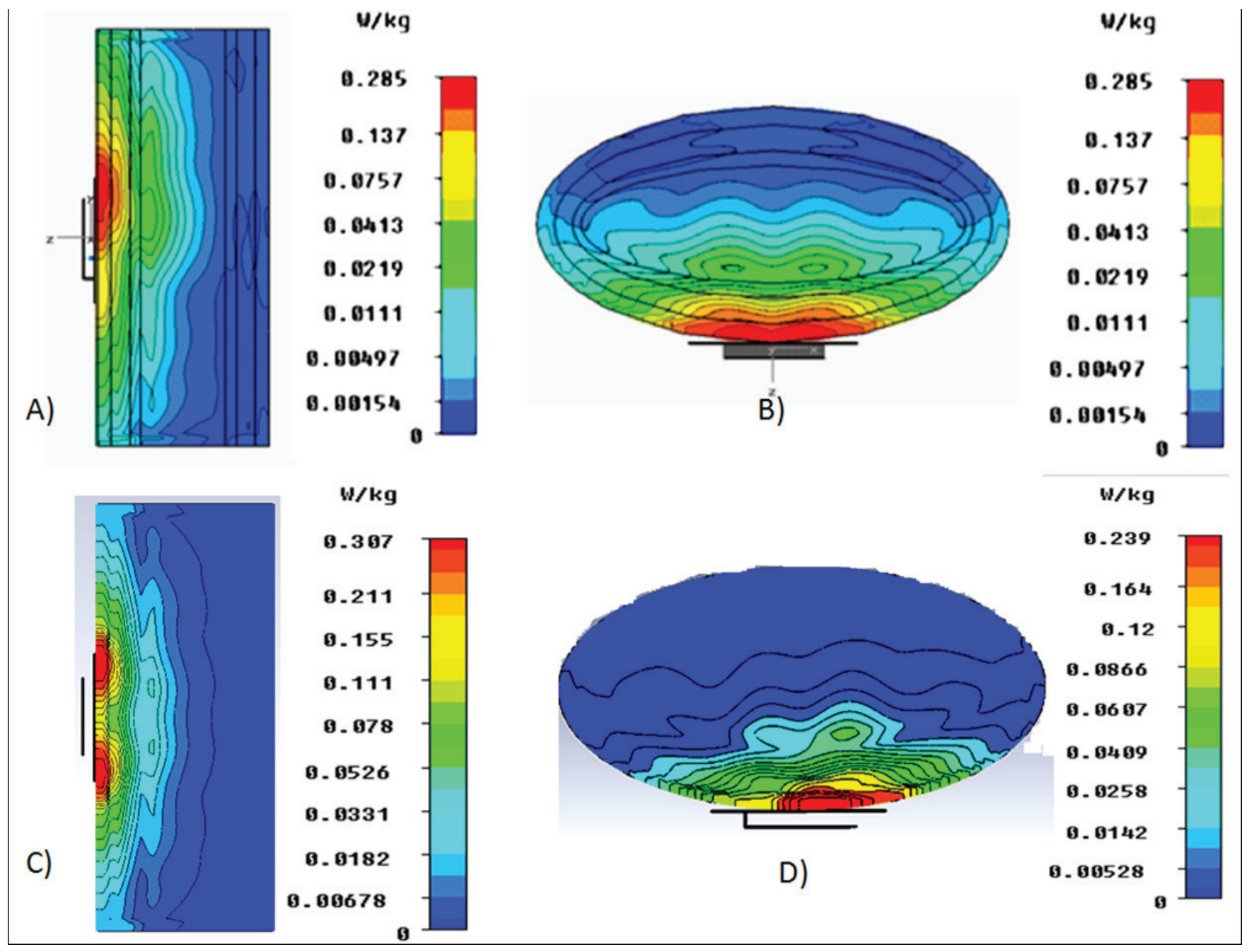

Figure 15. The Specific Absorption Rate (SAR) [W/Kg] of the human torso model wearing the reader's PIFA antenna for vertical polarization (Cases $A$ and $B$ ) and horizontal polarization (Cases C and D).

readers, as well as with conventional hand-held radios, and even with smart phones. Moreover, the continuous improvement in microchip sensitivity will permit extending the reading distance or, conversely, reducing the required powers. New pervasive applications may therefore be envisaged, where lowcost and even disposable wearable tags will interact with multiservice radio devices.

The position of the tags over the body has to be carefully chosen in order to avoid shadowing and excessive absorption. A single tag is not enough to establish an omnidirectional offbody link. Two or three tags placed over the chest, the shoulders, or over the arms permit interacting with the moving body from any angle and with remarkable reproducibility.

Finally, the backscattered power level in on-body configurations undergoes fluctuations with amplitudes of from $\pm 1 \mathrm{~dB}$ up to $\pm 4 \mathrm{~dB}$, depending on the position and on the motion. This issue has to be carefully taken into account in sensing applications based on RSSI processing. Such fluctuations may result in being synchronized to the movement of the arm to which the antenna is attached, revealing that selective motion detection could be possible by using very low-cost technology.

\section{Acknowledgments}

This research was supported by the Italian Ministry of University under funded project PRIN2008 "RFID MULTITAG." The authors would like to thank G. Contri, A. Giuliani, and M. Trecca for technical support of the measurements.

\section{References}

1. S. Jongwoo, T. Sanchez Lopez, and K. Daeyoung, "The EPC Sensor Network for RFID and WSN Integration Infrastructure," Fifth Annual IEEE International Conference on Pervasive Computing and Communications Workshops, March 2007.

2. L. Cheng-Ju, L. Li, C. Shi-Zong, W. Chi Chen, H. ChunHuang, and C. Xin-Mei, "Mobile Healthcare Service System Using RFID," IEEE International Conference Networking on Sensing and Control, 2, 2004, pp. 1014-1019.

3. R. S. Sangwan, R. G. Qiu, and D. Jessen, "Using RFID Tags for Tracking Patients, Charts and Medical Equipment Within 
an Integrated Health Delivery Network," IEEE International Conference Networking Sensing and Control, 2005, pp. 10701074.

4. J. Park, J. Seol, and Y. Oh, "Design and Implementation of an Effective Mobile Healthcare System Using Mobile and RFID Technology," Proceedings of the 7th International Symposium HEALTCOM, 2005, pp. 263-266.

5. Won Jay Song, S. H. Son, Munkee Choi, and Minho Kang, "Privacy and Security Control Architecture for Ubiquitous RFID Healthcare System in Wireless Sensor Networks," International Conference on Consumer Electronics, January 2006.

6. O. Rashid, P. Coulton, R. Edwards, and W. Bamford, "Utilizing RFID for Mixed Reality Mobile Games," IEEE International Conference on Consumer Electronics, January 2006.

7. G. Marrocco, "RFID Antennas for the UHF Remote Monitoring of Human Subjects," IEEE Transactions on Antennas and Propagation, AP-55, 6, June 2007, pp. 1862-1680.

8. C. Occhiuzzi, S. Cippitelli, and G. Marrocco, "Modeling, Design and Experimentation of Wearable RFID Sensor Tag," IEEE Transactions on Antennas and Propagation, AP-58, 8, August 2010, pp. 2490-2498.

9. L. Yang, R. Vyas, A. Rida, J. Pan, and M. M. Tentzeris, "Wearable RFID-Enabled Sensor Nodes for Biomedical Applications," Proceedings of Electronic Components and Technology Conference, Lake Buena Vista, Florida, 2008.

10. M. Polívka, M. Švanda and P. Hudec, "UHF RFID for People," Development and Implementation of RFID Technology, I-Tech Education and Publishing, 2009.

11. M. Polivka, M. Svanda, P. Hudec, and S. Zvanovec, "UHF RF Identification of People in Indoor and Open Areas," IEEE Transactions on Microwave Theory Techniques, 57, 5 Part 2, May 2009, pp. 1341-1347.

12. T. Kellomaki, T. Bjorninen, L. Ukkonen, and L. Sydanheimo, "Shirt Collar Tag for Wearable UHF RFID Systems," European Conference on Antennas and Propagation EUCAP, 2010.

13. Harish Rajagopalan and Y. Rahmat-Samii, "Conformal RFID Antenna Design Suitable for Human Monitoring and Metallic Platforms," European Conference on Antennas and Propagation EUCAP, 2010.

14. P. S. Hall and Y. Hao, Antennas and Propagation for BodyCentric Wireless Communications, Norwood, MA, Artech House, 2006.

15. J. Garcìa, A. Arriola, G. Sasiain, D. Valderas, J. I. Sancho, and X. Chen, "Characterization of Phantom Size and Link Budget for Off-Body Communications," European Conference on Antennas and Propagation EUCAP, 2010.
16. R. Khouri, P. Ratajczak, P. Brachat, and R. Staraj, "A Thin Surface-Wave Antenna Using a Via-Less EBG Structure for $2.45 \mathrm{GHz}$ On-Body Communication Systems," European Conference on Antennas and Propagation EUCAP, 2010.

17. L. Vallozzi, W. Vandendriessche, H. Rogier, C. Hertleer, and M. Scarpello, "Wearable Textile GPS Antenna for Integration in Protective Garments," European Conference on Antennas and Propagation EUCAP, 2010.

18. S. Subramaniam and B. Gupta, "A Bluetooth Antenna for On-Body Communications," European Conference on Antennas and Propagation EUCAP, 2010.

19. A. Sani, A. Alomainy, G. Palikaras, Y. Nechayev, C. Parini, and P. S. Hall, "UWB On-Body Radio Propagation and System Modeling for Wireless Body-Centric Networks," IEEE Transactions on Antennas and Propagation, AP-58, 1, January 2010, pp. 238-241.

20. F. Yang, A. Aminian, and Y. Rahmat Samii, "A Thin Surface-Wave Antenna Using a Via-Less EBG Structure for 2.45 $\mathrm{GHz}$ On-Body Communication Systems," European Conference on Antennas and Propagation EUCAP, 2010.

21. G. Marrocco, E. Di Giampaolo, and R. Aliberti, "Estimation of UHF RFID Reading Regions in Real Environments," IEEE Antennas and Propagation Magazine, 51, 6, December 2009, pp. 44-57.

22. D. Psychoudakis, G. Y. Lee, C-C. Chen, and J. L. Volakis, "Military UHF Body-Worn Antennas for Armored Vests," European Conference on Antennas and Propagation EUCAP, 2010.

23. C. Hertleer, L. Van Langenhove, H. Rogier, and L. Vallozzi, “A Textile Antenna for Fire Fighter Garments," AUTEX 2007 Conference (Association of Universities for Textiles): From Emerging Innovations to Global Business, 2007.

24. C. Occhiuzzi and G. Marrocco, "The RFID Technology for Neurosciences: Feasibility of Limbs' Monitoring in Sleep Diseases," IEEE Transactions on Information Technology in Biomedicine, 14, 2010, pp. 37-43.

25. D. D. Arumugam and D. W. Engels, "Specific Absorption Rates in the Human Head and Shoulder for Passive UHF RFID Systems at 915MHz," Proceedings the XXIX General Assembly of the International Union of Radio Science URSI, 2008.

26. G. Marrocco, L. Mattioni, and C. Calabrese, "Multi-Port Sensor RFIDs for Wireless Passive Sensing - Basic Theory and Early Simulations," IEEE Transactions on Antennas and Propagation, AP-56, 8, August 2008, pp. 2691-2702.

27. J. Zhao, Y. Zhang, and M. Ye, "Research on the Received Signal Strength Indication Location Algorithm for RFID System," International Symposium Communications and Information Technologies, Bangkok, October 2006, pp. 881-885. 
28. P. Salonen, Y. Rahmat-Samii, M. Schaffrath, and M. Kivikoski, "Effect of Textile Materials on Wearable Antenna Performance: A Case Study of GPS Antennas," IEEE International Symposium on Antennas and Propagation, 1, 2004, pp. 459-462.

29. P. Salonen and Y. Rahmat-Samii, "Textile Antennas: Effects of Antenna Bending on Input Matching and Impedance Bandwidth," Proceeding European Conference on Antennas and Propagation EUCAP, France, November 2006, pp. 1-5.

30. S. Z. Zhu and R. Langley, "Dual-Band Wearable Textile Antenna on an EBG Substrate," IEEE Transactions on Antennas and Propagation, AP-57, 3, April 2009, pp. 926-935.

31. http://www.nxp.com/documents/application_note/ AN173211.pdf.

32. G. Marrocco and F. Amato, "Self-Sensing Passive RFID: from Theory to Tag Design and Experimentation," 39th European Microwave Conference, 2009.

33. http://www.thingmagic.com/fixed-rfid-readers/mercury5.

34. A. Alomainy, Y. Hao, X. Hu, C. G. Parini, and P. S. Hall, "UWB On-Body Radio Propagation and System Modeling for Wireless Body-Centric Networks," Communications IEE Proceedings, 153, 1, 2006, pp. 107-114.

35. C. Occhiuzzi, A. Rida, G. Marrocco, and M. Tentzeris, "RFID Passive Gas Sensor Integrating Carbon Nanotubes," IEEE Transactions on Microwave Theory and Techniques, 99, August 2010.

36. D. D. Arumugam, D. W. Engels, and M. H. Mickle, "Specific Absorption Rates in Muscle Tissues for Passive UHF RFID Tag Backscatter," Radio and Wireless Symposium, January 2009, pp. 445-448.

\section{Introducing the Feature Article Authors}

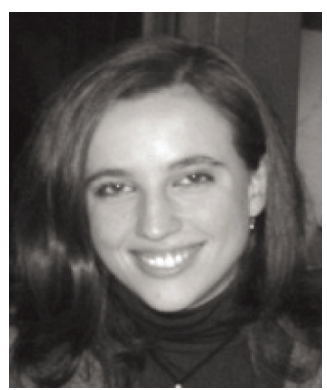

Sabina Manzari was born in Rome, Italy, in 1985. She received her MSc in Medical Engineering from the University of Rome "Tor Vergata" in 2010, where she is currently working as a grant researcher. Her research interests include electromagnetism, passive sensors, hypertermic treatments, and wireless health monitoring by means of wearable radio-frequency identification techniques. In 2010-2011, she was a Visiting Researcher in the RFID research group of Tampere
University of Technology (Finland). Her research was mainly focused on the development of passive RFID sensors for temperature and heat monitoring.

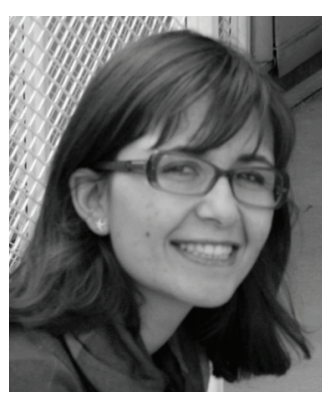

Cecilia Occhiuzzi received the MSc in Medical Engineering from the University of Rome "Tor Vergata," Rome, Italy. She is currently working toward the $\mathrm{PhD}$ in Electromagnetics (with an interest is wireless health monitoring by means of wearable and implantable RF identification techniques) at the University of Rome "Tor Vergata." In 2008, she was with the School of Engineering, University of Warwick, Warwick, $\mathrm{UK}$, as a postgraduate student involved with the design and implementation of wireless surface-acoustic-wave (SAW) sensors. In 2010, she was a Visiting Researcher with the Georgia Institute of Technology, Atlanta. She holds two patents on sensor RFID systems. Her research was mainly focused on the design of passive RFID sensors for structural health monitoring and gas detection by means of CNT-based tags

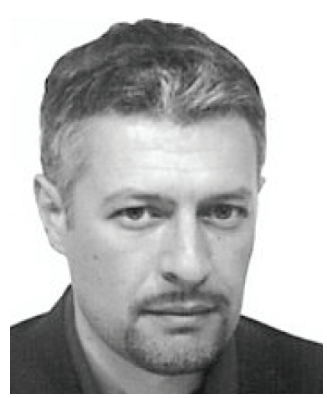

Gaetano Marrocco was born in Teramo, Italy, in 1969. $\mathrm{He}$ received the Laurea degree in Electronic Engineering (Laurea cum Laude and Academic Honour), and the $\mathrm{PhD}$ in Applied Electromagnetics, from the University of L'Aquila, Italy, in 1994 and 1998, respectively. Since 1997, he has been a Researcher at the University of Rome "Tor Vergata," Rome, Italy. He currently teaches antenna design and medical radio systems, manages the Antenna Lab and the RFID Lab, and is an advisor in the Geo-Information PhD program. In October 2010, he became an Associate Professor of Electromagnetics.

In 1994, he was at the University of Illinois at UrbanaChampaign as a postgraduate student. In 1999, he was a Visiting Researcher at the Imperial College in London, UK. In 2008, he joined the $\mathrm{PhD}$ program of the University of Grenoble, France. His research is mainly directed toward the modeling and design of broadband and ultra-wideband (UWB) antennas and arrays, as well as toward sensor-oriented miniaturized antennas for biomedicine, aeronautics, and radio-frequency identification 
(RFID). Prof. Marrocco has been involved in several space, avionic, naval, and vehicular programs of the European Space Agency, NATO, the Italian Space Agency, and the Italian Navy concern with the analysis and design of non-conventional antennas and systems over platforms. He holds eight patents on broadband naval antennas and structural arrays, and on sensor RFID systems.

He currently serves as Associate Editor of the IEEE Antennas and Wireless Propagation Letters, and as a member of the Technical Program Committees of IEEE RFID, IEEE IMS, and ISABEL. In 2008, he was the General Chair of the first Italian multidisciplinary scientific workshop on RFID: RFIDays-2008: Emerging Technology for Radiofrequency Identification. He was the co-chair of the RFIDays-2010 International Workshop in Finland, and Chair of the Local Committee of the V European Conference on Antennas and Propagation. In 2011, he was nominated Vice Chair of the Italian Commission D delegation of URSI. $4 \mathbf{A s}_{\mathrm{s}}^{\circ}$

\section{To All IEEE Life Members}

Life Member status in the IEEE is an earned honor. Each year, most Life Members are sent a Life Member Profile in lieu of a membership dues invoice. Life Members must return the Profile, indicating that they wish to keep their membership(s) active, even if there are no changes. If the profile is not returned, it is assumed that their membership should not be kept active, and they do not receive any complimentary Society publications to which they may be entitled. If applicable, the complimentary Society publications that a Life Member would like to continue receiving must be indicated on the Profile. Life Members can still retain their membership in a Society, even if they do not wish to receive its publications. However, they must return the Profile.

The Profile can also be updated online at www.ieee. org/renewal, or by logging into "myIEEE" at http://www. ieee.org/, using an IEEE Web account. Those not having an IEEE Web account can register for one at www.ieee.org/ web/accounts/. 